\title{
NOTE ON THE DIFFERENTIAL INVARIANTS OF A SURFACE
}

\author{
AND OF SPACE*
}

BY

\section{CHARLES NELSON HASKINS}

Forsyth $\dagger$ has recently discussed at some length the construction and the geometric meaning of the differential invariants of a surface and of space, i. e., the differential invariants of binary quadratic differential forms and of ternary quadratic differential forms of class zero.

It may not be amiss to point out that the methods of Riccr's $\ddagger$ Absolute Differential Calculus render unnecessary much of the complicated calculation which occupies so large a part of Forsytr's memoirs on this subject, and which, in his opinion $\S$ offers an insuperable obstacle to further progress by the methods he has used.

The problem of the determination of the invariants of quadratic differential forms consists of two parts : first, the reduction of the problem to one in the theory of the projective invariants of algebraic forms; and second, the solution of the problem thus reduced. Now the first and more difficult of these two problems has been solved once for all by the introduction by Christoffel $\|$ and RICCI $\$ of the process of covariantive differentiation. ForsYTH has recognized the double nature of the problem, and has devoted a considerable portion of his memoirs on the subject to the solution of the firet part as a necessary prelude to the investigation of the second part. This solution, by means of partial differential equations, he has carried out with much skill, although hampered by the use of the wholly unsuitable notation of ZoRAwskI* which effectually conceals the greater part of the symmetry of the problem. The result of this part of his work is the introduction of new variables which differ but

* Presented to the Society at the Williamstown summer meeting September 8, 1905 . Received for publication October 1, 1905.

† Forsyth, Philosophical Transactions, series A, vol. 201 (1903), pp. 329-403; series A, vol. 202 (1903), pp. 277-333.

$\ddagger$ Ricot and Levi-Crvita, Mathematisohe Annalen, vol. 54 (1900), pp. 125-201.

§ ForsYTH, loo. cit., vol. 202, p. 304.

\|Christoffal, Crelle's Journal, vol. 70 (1869), p. 46.

II Ricci and Levi-Civita, loc. cit., p. 138.

* Zorawski, Aota Mathematica, vol. 16 (1892), p. 1. 
slightly from the multiple-index symbols of Christofrel and the covariant derivatives of Ricci.

This result could, hovever, have been foreseen, and the reduction to the algebraic problem immediately written down, by making use of a theorem due to Ricci. *

As to the details of ForsyTh's work the following may be said:

(1) The fundamental magnitudes $P, Q, R, S ; \alpha, \beta, \gamma, \delta, \epsilon \nmid$ are simple combinations of covariant derivatives of the fundamental magnitudes $L, M, N$. The new relations $\ddagger$ between the magnitudes $L, M, N ; P, Q, R, S$ and the quantities $E, F, G$ and their derivatives are but the first of a series of such relations, infinite in number, which are directly obtainable by successive covariantive differentiation of the relation of Gauss,

$$
\frac{(12,12)}{a}=\frac{L N-M^{2}}{E G-F^{2}} \quad\left(a \equiv E G-F^{2}\right) .
$$

The invariant of order three which ForsyTh $\S$ obtains as a solution of twentyeight partial differential equations is, if use is made of the five-index symbols $(12,121),(12,122)$, immediately obtainable as an invariant of the forms \|

$$
\begin{gathered}
(E, F, G)(*)^{2}, \\
((12,121),(12,122))(*)^{1} .
\end{gathered}
$$

(2) In the determination of the invariants, or rather differential parameters, of the third order for the ternary form, Fonsytr introduces new variables $a^{\prime \prime}$, etc., which are closely related to the covariant derivatives of the function $\phi$. The resulting equations are then simplified by the use of the fact that the fourindex symbols, or the quantities $\Theta_{1}, \ldots, \Theta_{6}$, which differ from them by the factor

$$
4\left|\begin{array}{lll}
a & h & g \\
h & b & f \\
g & f & c
\end{array}\right|
$$

only, vanish because the form is of class zero, $i$. e., reducible to the type $d x^{2}+d y^{2}+d z^{2} \pi$. Had the covariant derivatives instead of the functions $\mathrm{a}^{\prime \prime}$, etc., been used this restrictive simplification would have been unnecessary.

* Ricci and Levi-Civita. loc. cit., p. 162.

† Forsyth, loo. cit., vol. 201, p. 334.

† FORSYTH, loc. cit., vol. 201, p. 401.

§ ForsYTH, loo. cit., vol. 201, p. 399.

\|Cf. Haskins, these Transactions, vol. 3 (1902), p. 87. The invariant in question is a solution of the four equations (28) there given.

If Forsytr, loo. cit., vol. 202, p. 317. 
ForsyTh's work on the ternary form in so far as it relates to the reduction of the problem to one in algebraic forms, has been wholly anticipated by RICCI* who has shown that for the unrestricted form, the invariants and parameters sought are invariants of the system consisting of the fundamental form $d s^{2}$, the forms whose coefficients are the covariant derivatives of $\phi$, and a form whose coefficients $\alpha_{r e}$ form the covariant system reciprocal to the contravariant system

NEW BEDFORD, MA88,

$$
\alpha^{(r s)} \equiv \frac{(r+1, r+2, s+1, s+2)}{\left|a_{11} a_{22} a_{33}\right|} .
$$

$$
\text { August 27, } 1905 .
$$

* Ricci and Levi-Civits, loo. cit., pp. 162, 163. 